\title{
Observations and Models for Needle-Tissue Interactions
}

\author{
Sarthak Misra, Kyle B. Reed, Benjamin W. Schafer, K. T. Ramesh, and Allison M. Okamura
}

\begin{abstract}
The asymmetry of a bevel-tip needle results in the needle naturally bending when it is inserted into soft tissue. In this study we present a mechanics-based model that calculates the deflection of the needle embedded in an elastic medium. Microscopic observations for several needlegel interactions were used to characterize the interactions at the bevel tip and along the needle shaft. The model design was guided by microscopic observations of several needlegel interactions. The energy-based model formulation incorporates tissue-specific parameters such as rupture toughness, nonlinear material elasticity, and interaction stiffness, and needle geometric and material properties. Simulation results follow similar trends (deflection and radius of curvature) to those observed in macroscopic experimental studies of a robotdriven needle interacting with different kinds of gels. These results contribute to a mechanics-based model of robotic needle steering, extending previous work on kinematic models.
\end{abstract}

\section{INTRODUCTION}

Percutaneous needle insertion is one of the most common minimally invasive clinical procedures. It is used for diagnosis, localized therapeutic drug delivery, and tissue sample removal from tumors deep within the body. For effective medical diagnoses and treatments, the needle must reach its intended target. However, tissue inhomogeneity and anisotropy, organ deformation, anatomy obstructing needle path, and physiological processes, such as respiration, flow of fluids, and edema cause the needle to deviate from its intended path. A possible method to mitigate needle targeting errors is to use a needle that can be robotically steered inside the body to reach the intended target.

Several groups have examined the use of robotically steered flexible needles through tissue [1], [2], [3], [4], [5], [6], [7]. Abolhassani et al. [8] provide a summary of recent research being conducted in the area of robotic needle insertion in soft tissue. Planning such procedures requires an accurate model of the needle-tissue interaction. A general survey of needle and tissue interaction models which describes both physics- and non-physics-based interaction models are provided in [9]. Further, experimental work has identified forces (due to puncture, cutting, and friction) that develop during needle insertion through tissue [10]. In [7], a kinematic model specifically for bevel-tip needle steering was presented whose parameters were fit using experimental data. However, this model did not consider the interaction of the needle with an elastic medium.

This work was supported by National Institutes of Health grant (R01 EB006435) and a Link Foundation fellowship. S. Misra, K. B. Reed, K. T. Ramesh, and A. M. Okamura are with the Department of Mechanical Engineering, The Johns Hopkins University. B. W. Schafer is with the Department of Civil Engineering, The Johns Hopkins University. \{sarthak, reedkb, schafer, ramesh, aokamura\} @ jhu.edu

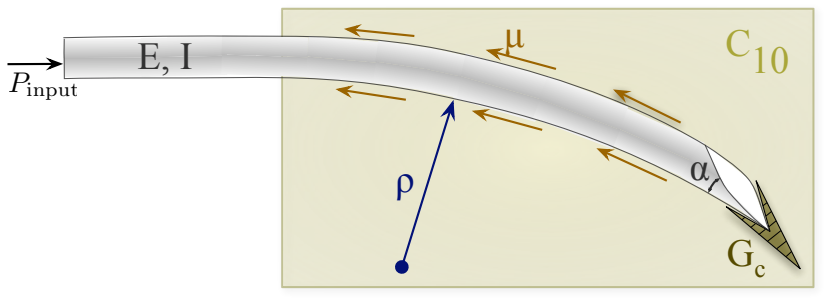

Fig. 1. Schematic of a bevel-tip needle interacting with a soft elastic medium. The two dimensional model incorporates tip forces generated by rupture, tissue properties (toughness: $G_{C}$, nonlinear elasticity: $C_{10}$ ) and needle properties (bevel angle: $\alpha$ and flexural rigidity: $E I$ ).

In this study, we present a two-dimensional model for a bevel-tip needle embedded in an elastic medium, shown in Fig. 1. The mechanics-based model is based on both microscopic observations of the needle tip interacting with soft gels and macroscopic observations of a robotically steered needle inserted through various gels. The model accounts for the needle's geometric and material properties, and also the medium's nonlinear material properties. In addition to capturing needle tip effects, our model attempts to provide a physics-based understanding of the effect of the needle-tissue interaction stiffness on the evolving shape of the needle.

\section{OBSERVATIONS OF NEEDLE-TISSUE INTERACTIONS}

In order to develop models for a needle embedded in an elastic medium, we conducted micro- and macroscopic experiments. Nitinol wires (Nitinol Devices and Components, Fremont, CA, USA) of varying diameter were used as flexible needles. Each needle had a bevel tip. A smooth and sharp bevel was obtained by fixing the needles at an angle in Crystalbond $^{\mathrm{TM}}$ (Aremco Products, Inc., Valley Cottage, NY, USA) mounting adhesive, which is a thermo-plastic, and then polishing the edges. Plastisol (M-F Manufacturing Co., Inc., Ft. Worth, TX, USA) gels were used in the experiments; the ratio of plastic and softener was varied to change the stiffness of the gel.

\section{A. Microscopic Observations}

Microscopic observations of needle and elastic medium interactions have been reported previously (e.g., [11]), but most of the published literature focuses on observing the damage to the gel or tissue surface after the needle has punctured the medium. Sections of the gel have also been observed, but again only after the needle has penetrated the medium. In order to observe the interaction within the gel as the needle is embedded in the medium, we used a Zeiss LSM 510 Meta laser scanning confocal microscope. The needle and gel were visualized with differential interference contrast 

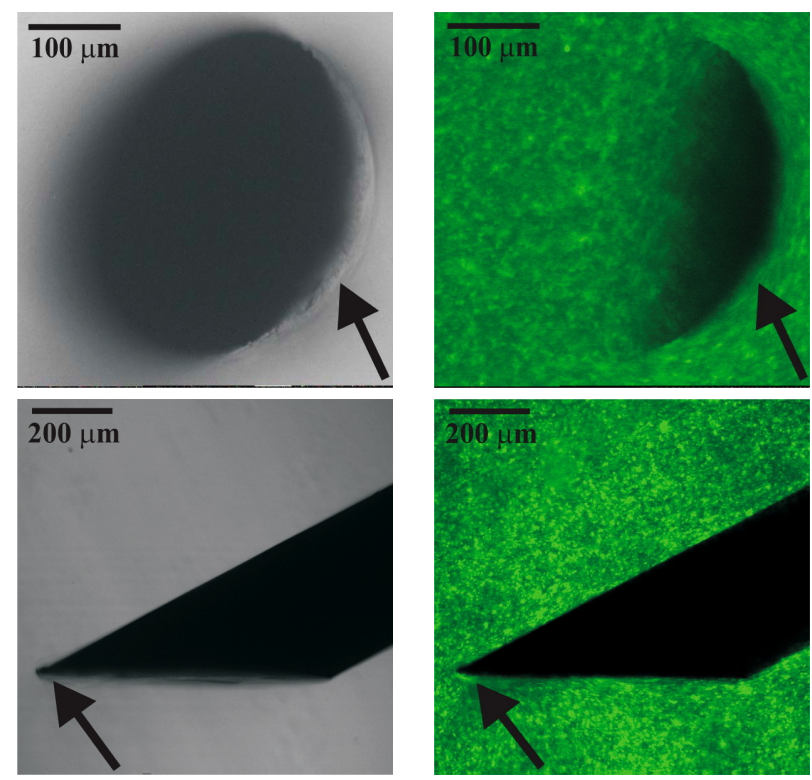

(a)
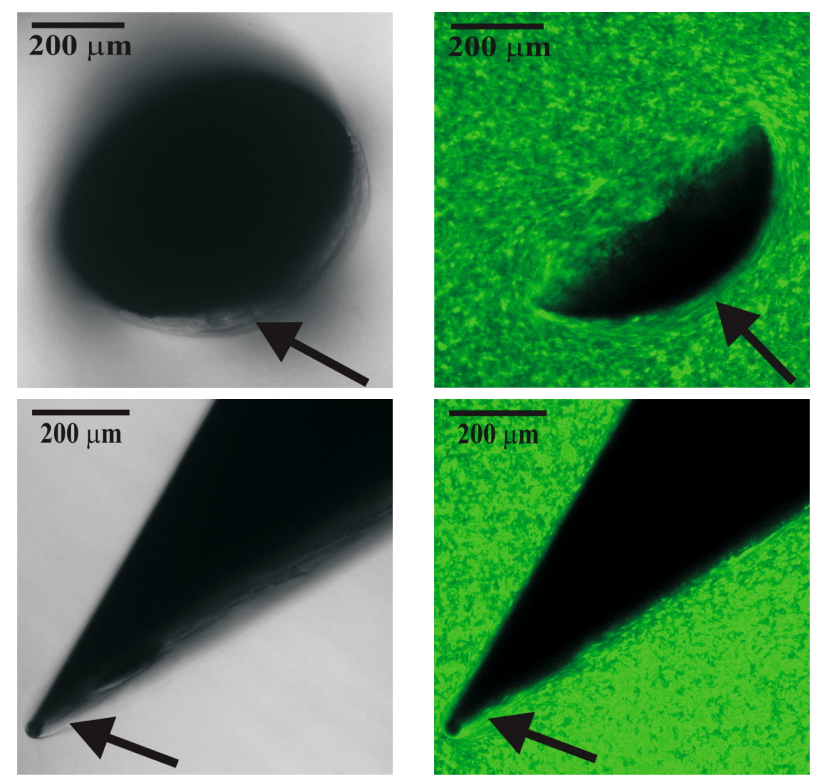

(b)

Fig. 2. Example DIC and epifluorescent images taken using a confocal microscope where the first row are in the axial configuration, while the second row pertain to the perpendicular configuration. (a) $\varnothing 0.38 \mathrm{~mm}, \alpha=26.4^{\circ}$ (b) $\varnothing 0.71 \mathrm{~mm}, \alpha=28.2^{\circ}$. Rupture of the gel is observed close to bevel edge of the needle, while the gel compresses against the needle shaft and the bevel face of the needle tip.

(DIC), epifluoresence, and reflected light using the $488 \mathrm{~nm}$ line of an argon/ion laser and 0.3 NA Plan-Neofluar 10x objective lenses (Carl Zeiss Inc., Maple Grove, MN, USA) with pin hole diameter set at 9.33 Airy units.

The ratio of plastic to softener for Plastisol gel was 4:1. The Plastisol gel $\left(400 \mathrm{~cm}^{3}\right)$ was doped with $20 \mu \mathrm{l}$ of $10 \mathrm{mg} / \mathrm{ml}$ rhodamine green solution (Invitrogen, Carlsbad, CA, USA). This dye was added to facilitate epifluorescent confocal imaging of Plastisol gel. Since a very small amount of dye was used, we assume that it does not significantly change the material properties of the gel. Cubes of $0.5 \mathrm{~cm}^{3}$ were prepared and needles were manually inserted into the gel and viewed under the microscope. Observations were made in two configurations (axial and perpendicular), for needles of 4 different diameters and bevel angles. In the axial configuration, the laser light was along the needle axis, while during the perpendicular observation the needle-gel sample was configured such that the laser light was perpendicular to the needle axis. DIC and epifluorescent images were obtained for each configuration, two sets are shown in Fig. 2.

For all the needles, rupture or tearing is observed at the bevel edge, while compression is observed along the needle shaft. Rupture is observed in the DIC images while compression is seen in the epifluorescent images. In the $a x$ ial configuration, the needle cross-section appears crescentshaped because of the bevel edge. In the perpendicular configuration, compression due to the needle tip pushing against the gel is observed, and also slight rupture is seen at the needle tip in the DIC images. Unlike brittle materials, crack propagation (classical Mode-I fracture) is not seen during the interaction of sharp needles and soft gels.

\section{B. Macroscopic Observations}

We used the setup described in [12] to measure the radius of curvature for three needles inserted into two phantom tissues. The device can rotate and insert a needle into phantom tissues, and measure the forces and torques at the base of the needle. Nitinol wires with diameters of $0.40 \mathrm{~mm}$ $\left(\alpha=38.0^{\circ}\right), 0.71 \mathrm{~mm}\left(\alpha=38.7^{\circ}\right)$, and $0.90 \mathrm{~mm}\left(\alpha=30.9^{\circ}\right)$ were used. The two transparent Plastisol tissues have a plastic to softener ratio of 4:1 (gel \#1) and 32:9 (gel \#2). Tracking was performed at $7.5 \mathrm{~Hz}$ by triangulating images from a pair of XCD-X710 firewire cameras (Sony Corporation, Tokyo, Japan) mounted above the phantom tissue.

For each experiment, we inserted the needle $20 \mathrm{~cm}$ into the gel at a constant velocity of $0.25 \mathrm{~cm} / \mathrm{sec}$. To determine the radius of curvature throughout the insertion, we segmented the needle tip position every $5 \mathrm{~cm}$ and calculated the radius of curvature over three regions. The first $5 \mathrm{~cm}$ were not used in the radius of curvature calculation because the pre-puncture deformation, the interaction of the support sheath, and the gel surface contaminate the initial needle deflection data. Fig. 3 shows the needle tip positions for three needles inserted into both materials. The figures only show planar data since the depth direction only varied by a maximum of $15 \mathrm{~mm}$. The radius of curvature for each insertion was determined using a least squares three-dimensional fitting algorithm. Table I shows the radii calculated over each section of the tissue. The radius of curvature varies by an average of $10.7 \%$ for these six insertions with most of this deviation found in the $\varnothing 0.90 \mathrm{~mm}$ needle. The other two needles only have an average deviation of $5.7 \%$. Given this low variation, we assume a constant radius of curvature throughout the insertion, which corresponds to the kinematic model of [7]. 


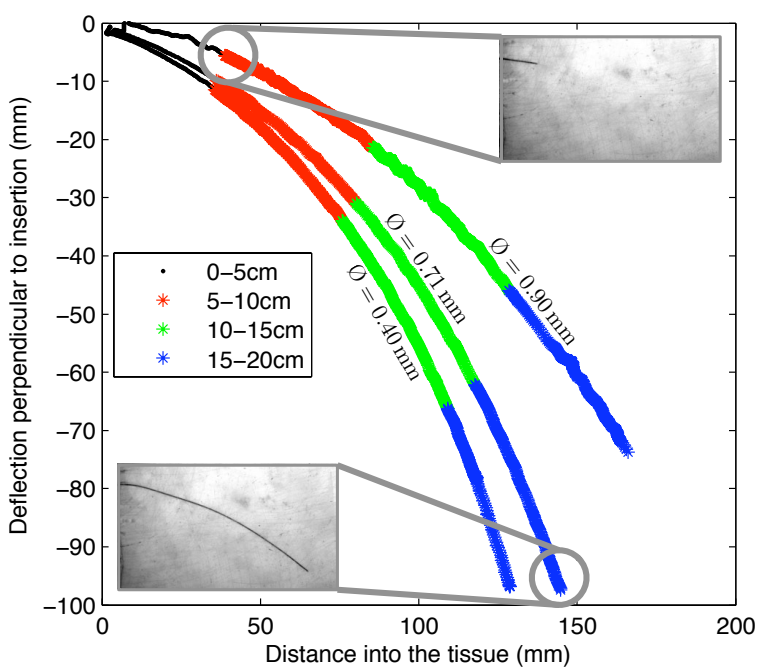

(a)

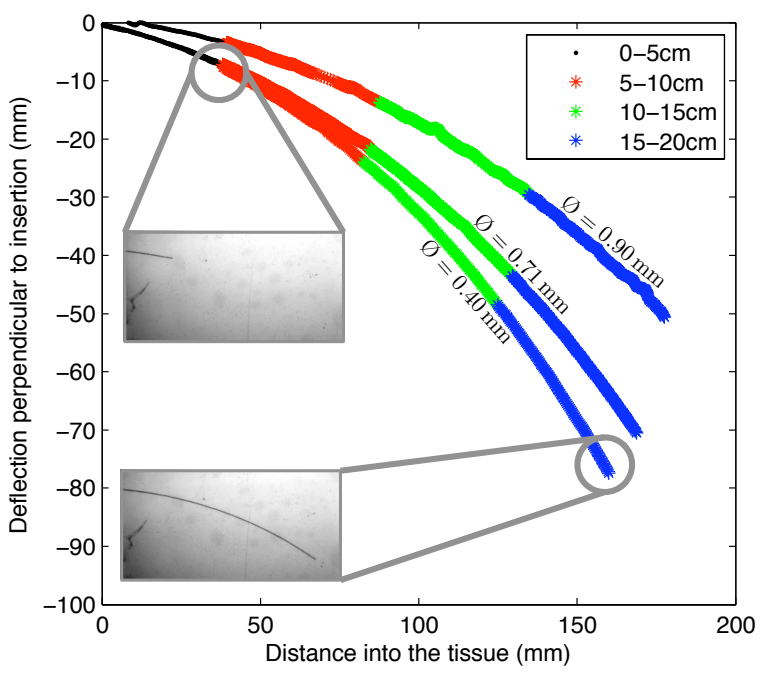

(b)

Fig. 3. Needle tip position: During each $20 \mathrm{~cm}$ insertion, the tip position is measured. The radius of curvature remains relatively constant throughout the insertion. (a) Gel one (stiffest). (b) Gel two (less stiff).

\section{MOdElS FOR NEEDLE-TISSUE INTERACTION}

We now present mechanics-based models that incorporate and capture the observations from micro- and macroscopic experiments described in the previous section. We derive expressions that describe the deflection of a bevel-tip needle embedded in an elastic medium. The analysis assumes a twodimensional model with the needle undergoing a single bend. The derived expression incorporates the needle material and geometric properties, as well as the local and global elastic properties of the medium. Further, given the deflection, the radius of curvature of the needle can be readily evaluated. In the derivation presented below, the needle trajectory is discretized into a series of steps, $i$, where the length of the needle is $l_{i}$ and the deflection is $y_{i}$. Fig. 4 depicts the load distribution of a needle of length $l_{i}$ surrounded by an elastic medium, where $P_{\text {input }}$ is the applied needle insertion force, $P$ and $Q$ are the tip loads at the bevel edge, and $K_{T}$ is defined
TABLE I

RADIUS OF CURVATURE FOR NEEDLE PATH SEGMENTS

\begin{tabular}{|c|c|c|c|c|c|}
\hline gel, $\varnothing(\mathrm{mm})$ & $\begin{array}{c}5-10 \mathrm{~cm} \\
(\mathrm{~mm})\end{array}$ & $\begin{array}{c}10-15 \mathrm{~cm} \\
(\mathrm{~mm})\end{array}$ & $\begin{array}{c}5-20 \mathrm{~cm} \\
(\mathrm{~mm})\end{array}$ & $\begin{array}{c}\text { mean } \rho \\
(\mathrm{mm})\end{array}$ & $\begin{array}{c}\text { max \% } \\
\text { deviation }\end{array}$ \\
\hline$\# 1,0.40$ & 165.1 & 161.0 & 168.5 & 179.4 & $6.5 \%$ \\
\hline$\# 1,0.71$ & 201.0 & 176.8 & 191.5 & 196.6 & $5.9 \%$ \\
\hline$\# 1,0.90$ & 299.4 & 191.3 & 259.9 & 289.1 & $15.2 \%$ \\
\hline$\# 2,0.40$ & 278.1 & 264.2 & 272.8 & 276.0 & $2.0 \%$ \\
\hline$\# 2,0.71$ & 368.9 & 314.8 & 337.0 & 327.3 & $9.5 \%$ \\
\hline$\# 2,0.90$ & 541.7 & 340.2 & 428.7 & 404.1 & $26.4 \%$ \\
\hline
\end{tabular}

as the stiffness per unit length of the elastic medium. The functional form for the deflection of the needle in an elastic medium is initially assumed and the Rayleigh-Ritz approach is used to evaluate the coefficients of the deflection equation. The Rayleigh-Ritz method is a variational method in which the minimum of a potential defined by the sum of the total energy and work done by the system are calculated. The system potential, $\Lambda$, of a needle, $l_{i}$, interacting with an elastic medium, is given by

$$
\Lambda=\underbrace{\left(N_{E}+S_{E}\right)}_{\text {energy }}+\underbrace{\left(-W_{Q}-W_{P}-W_{R}\right)}_{\text {work }}+\underbrace{P_{\text {input }} l_{i}}_{\text {input work }},
$$

where $N_{E}$ and $S_{E}$ are the energies associated with needle bending and needle-tissue interaction, respectively, and $W_{Q}$ and $W_{P}$ are the work due to transverse and axial bevel tip loads, respectively, and $W_{R}$ is the work done to rupture the tissue. We now derive explicit expressions for each of the terms in (1).

\section{A. Needle Bending}

$N_{E}$ is the sum of energy due to pure needle bending, $U_{B}$, and bending due to axial load, $U_{P}$, i.e. $N_{E}=U_{B}+U_{P}$. The expression for curvature is

$$
\frac{1}{\rho}=\left(\frac{d^{2} y_{i} / d x^{2}}{\left(1+\left(d y_{i} / d x\right)^{2}\right)^{3 / 2}}\right) \approx \frac{d^{2} y_{i}}{d x^{2}}
$$

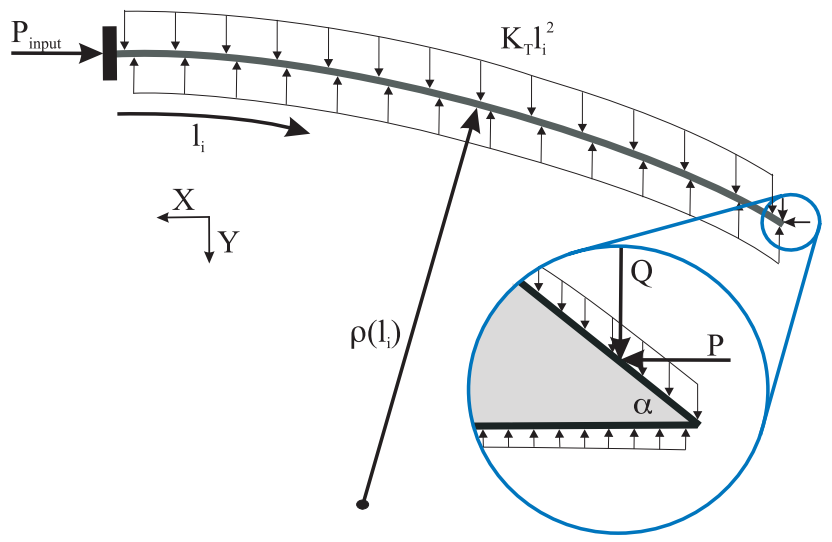

Fig. 4. Distributed load acting on a needle shaft as it interacts with an elastic medium. Inset: Forces acting on the bevel tip, where $P$ and $Q$ are the resultant forces along the bevel edge. $K_{T}$ is the stiffness of the elastic medium per unit length, while $P_{\text {input }}$ is the insertion force. 


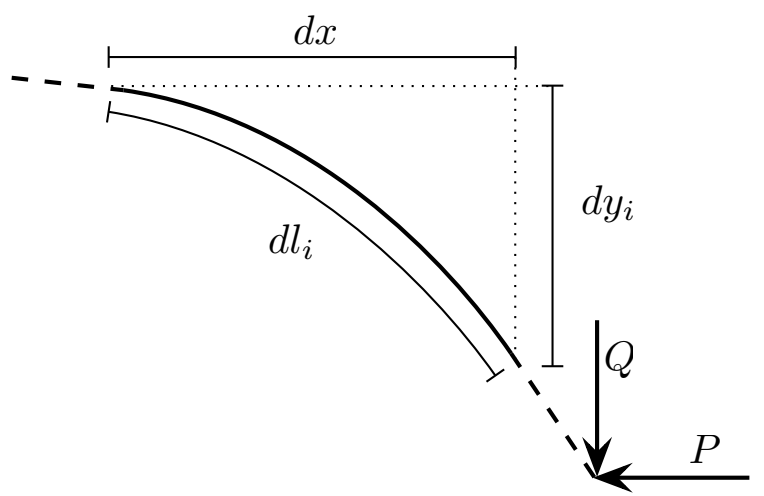

Fig. 5. Needle of segment $d l_{i}$ and with needle tip loads $P$ and $Q$.

Thus, for needle segments of length $l_{i}, N_{E}$ is reported in [13]

as

$$
U_{B}=\frac{E I}{2} \int_{0}^{l_{i}} \frac{1}{\rho^{2}} d x=\frac{E I}{2} \int_{0}^{l_{i}}\left(\frac{d^{2} y_{i}}{d x^{2}}\right)^{2} d x .
$$

The bending due to axial load, $P$, is evaluated by considering a segment, $d l_{i}$ (Fig. 5), along the needle. The axial strain due to $P$ is $\frac{d l_{i}}{d x}$. Thus, $E \frac{d l_{i}}{d x}=\frac{P}{A}$, where $A$ is the needle crosssectional area. Hence,

$$
U_{P}=\frac{1}{2} \int_{0}^{l_{i}} P d s=\frac{1}{2 A E} \int_{0}^{l_{i}} P^{2} d x .
$$

Substituting (3) and (4) into the expression for $N_{E}$ gives

$$
N_{E}=\underbrace{\frac{E I}{2} \int_{0}^{l_{i}}\left(\frac{d^{2} y_{i}}{d x^{2}}\right)^{2} d x}_{U_{B}}+\underbrace{\frac{1}{2 A E} \int_{0}^{l_{i}} P^{2} d x}_{U_{P}} .
$$

\section{B. Needle-Tissue Interaction}

In (1), $S_{E}$ is a combination of energy stored in the system due to compression of the elastic medium at the needle tip, $U_{C}$, and interaction of the elastic medium along the needle shaft, $U_{T}$. Thus,

$$
S_{E}=U_{C}+U_{T} .
$$

The compression of the gel due to the bevel-tip needle was observed both at the needle tip and along the needle shaft in microscopic images, as described in Section II-A. Pressure is applied by the needle and $\Delta V$ is the resulting change in volume of the elastic medium. Thus, $U_{C}$ is

$$
U_{C}=\text { Pressure } \times \Delta V=K\left(\frac{\Delta V}{V}\right)^{2},
$$

where $K$ is the bulk modulus of the elastic medium and the change in volume of the medium is $\Delta V=A l_{i}$. For linear elastic materials $K=\frac{E_{T}}{3\left(1-2 v_{T}\right)}$, where $E_{T}$ and $v_{T}$ are the Young's Modulus and Poisson's ratio of the medium, respectively. In order to express $U_{C}$ in terms of hyperelastic material parameters, consider the Cauchy stress, $\sigma_{22}=\frac{2 C_{10}}{\lambda}\left(\lambda^{3}-1\right)$, for a body under uniaxial compression, as derived in [14]. Evaluating the slope, $\frac{\partial \sigma_{22}}{\partial \lambda}$, for unit uniaxial stretch ratio, $\lambda=1$, results in $E_{T}=6 C_{10}$. Thus,

$$
U_{C}=\frac{2 C_{10}\left(A l_{i}\right)^{2}}{V\left(1-2 v_{T}\right)} .
$$

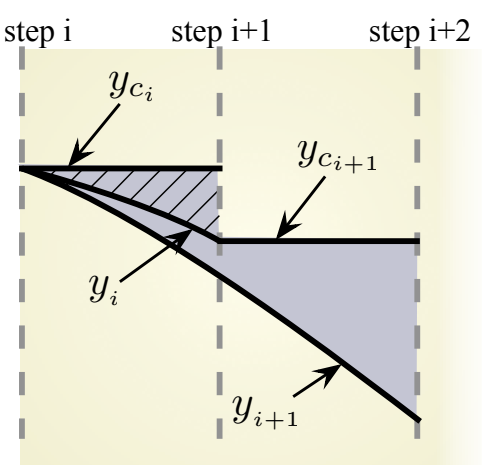

Fig. 6. Schematic for calculation of $U_{T}$, energy due to the interaction between the needle shaft and elastic medium.

As mentioned earlier, the needle trajectory is discretized into a series of steps and $U_{T}$ is calculated at each step. In the absence of an elastic medium surrounding the needle, no tip forces would be generated and the needle would not bend. This corresponds to the stress-free path of the needle and the deflection in this case is denoted by $y_{c_{i}}$. The key idea in the calculation of $U_{T}$ is that the elastic medium interacts with the needle shaft only when the needle deviates from the rupture trajectory it has previously established. Fig. 6 provides a sketch of $y_{c_{i}}$ and $y_{i}$, and the shaded area represents $U_{T}$ at each step. Thus, for $i=1$ and a needle segment of length $l_{1}$,

$$
U_{T}=\frac{1}{2} \int_{0}^{l_{1}} K_{T}\left(y_{1}-y_{c_{1}}\right)^{2} d x,
$$

where $K_{T}$ is the stiffness of the elastic medium per unit length and is calculated from the force-displacement or stress-strain curve of a tested sample (height: $h$, width: $w$, and depth: $d$ ) of the elastic medium. For an elastic medium $K_{T}=E_{T} \frac{d}{h}=6 C_{10} \frac{d}{h} \cdot y_{c_{1}}$ is a function of the bevel angle, $\alpha$, and for simplicity is assumed to be

$$
y_{c_{1}}=0 \text {. }
$$

Similarly for the needle segment of length, $l_{2}, U_{T}$ is calculated as the sum of energies for needle segments 0 to $l_{1}$ and $l_{1}$ to $l_{2}$

$$
U_{T}=\frac{1}{2} \int_{0}^{l_{1}} K_{T}\left(y_{2}-y_{c_{1}}\right)^{2} d x+\frac{1}{2} \int_{l_{1}}^{l_{2}} K_{T}\left(y_{2}-y_{c_{2}}\right)^{2} d x,
$$

where

$$
y_{c_{2}}=y_{1}\left(l_{1}\right)
$$

Thus, the general expression for $U_{T}$ for a needle of length, $l_{n}$, is

$$
U_{T}=\sum_{i=1}^{n} \frac{1}{2} \int_{0}^{l_{i}} K_{T}\left(y_{n}-y_{c_{i}}\right)^{2} d x,
$$

where

$$
y_{c_{i}}=y_{i-1}\left(l_{i-1}\right) .
$$

The necessity of treating $U_{T}$ step-wise can be understood through closer inspection of (13). The needle-tissue interaction energy is a function of the square of the deformation 
in the medium itself. If the needle was embedded in the tissue straight and then bent by tip loads, the deflection in the tissue would be $y_{i}$ itself. However, since the needle tears a path through the tissue, only the difference between the ruptured path and the current needle trajectory, i.e. $y_{i}-y_{c_{i}}$ interacts with the tissue. As insertion continues, the needle trajectory varies from the original ruptured path it has taken, thus engaging the tissue. Prediction of this process is handled step-wise; the previous path of the needle and current predicted path of the needle are known for every $i^{\text {th }}$ step. $y_{c_{i}}$ is a linear discretization of the ruptured path employed to determine the tissue deformation. Note, the path predicted by $y_{c_{i}}$ converges for smaller step sizes.

\section{Work Done}

In (1) the work done due to the transverse tip load, $Q$ is

$$
W_{Q}=Q y\left(l_{i}\right) .
$$

The work done by axial tip load, $P$, is

$$
W_{P}=\int_{0}^{l_{i}} P d u
$$

where $d u=d l_{i}-d x$, shortening of the needle, as shown in Fig. 5. Using the series expansion it is possible to express $d u$ in terms of $d y_{i}$ and $d x$

$$
d u=d l_{i}-d x=\sqrt{(d x)^{2}+\left(d y_{i}\right)^{2}}-d x \approx \frac{1}{2}\left(\frac{d y_{i}}{d x}\right)^{2} d x .
$$

Substituting (17) into (16) results in

$$
W_{P}=\int_{0}^{l_{i}} \frac{P}{2}\left(\frac{d y_{i}}{d x}\right)^{2} d x .
$$

The work done to rupture the elastic medium, $W_{R}$, is a function of the effective rupture toughness, $G_{C}$, and the amount of tear or rupture, $a . G_{C}$ is defined as the work done per unit needle cross-section area to rupture and cut through tissue [14]. Thus,

$$
W_{R}=a G_{C} l_{i}
$$

\section{Results}

We now present the needle deflection simulation results obtained using the model developed in the previous section. As mentioned earlier, in the Rayleigh-Ritz method, the functional form of the needle deflection is initially assumed. We chose

$$
y_{i}=a_{0}+a_{1} x+a_{2} x^{2}+a_{3} x^{3},
$$

where $y_{i}$ satisfies the boundary conditions $y_{i}(0)=0$ and $\frac{d y_{i}(0)}{d x}=0$. Using the Rayleigh-Ritz method, the coefficients $a_{0}, a_{1}, a_{2}$, and $a_{3}$ are evaluated by minimizing the system

TABLE II

SIMULATION INPUT PARAMETERS

\begin{tabular}{|c|c|c|c|c|c|}
\hline Needle & $\varnothing(\mathrm{mm})$ & $I\left(\mathrm{~m}^{4}\right)$ & $A\left(\mathrm{~mm}^{2}\right)$ & $E(\mathrm{GPa})$ & $a(\mathrm{~mm})$ \\
\hline & 1.0 & 0.05 & 0.79 & 50.0 & 0.05 \\
\hline \hline Tissue & $K_{T}(\mathrm{kN} / \mathrm{m})$ & $v$ & $C_{10}(\mathrm{kPa})$ & $G_{C}(\mathrm{~N} / \mathrm{m})$ & $V\left(\mathrm{~m}^{3}\right)$ \\
\hline & 25.0 & 0.45 & 4.0 & 20.0 & 0.0045 \\
\hline
\end{tabular}

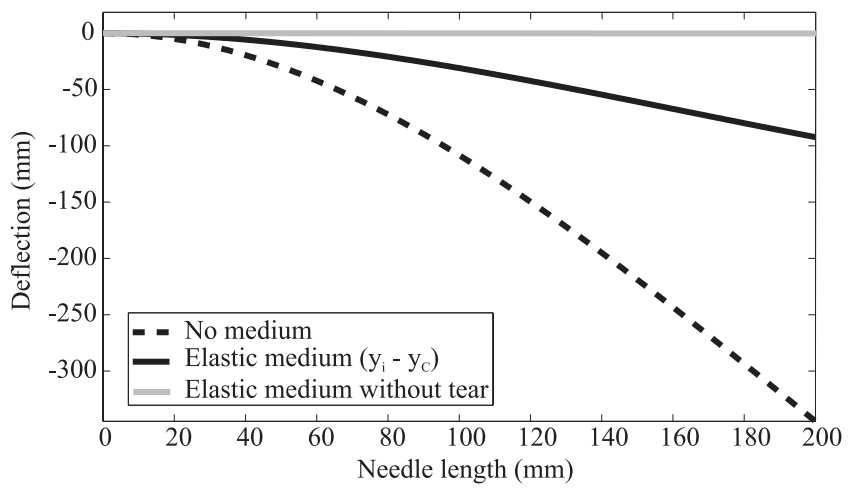

Fig. 7. Simulation result for needle deflection versus insertion.

potential, $\Lambda$ (1). For each step of needle length segment, $l_{i}, N_{E}, S_{E}, W_{Q}, W_{P}$, and $W_{R}$ are calculated using (5), (6), (15), (18), and (19), respectively. The deflection equation coefficients, $a_{j}$ (for $j=0,1,2,3$ ), are calculated by setting

$$
\frac{\partial \Lambda}{\partial a_{j}}=0 \text {. }
$$

The system parameters used for the simulation study are provided in Table II and are based on mean values calculated in [14].

Fig. 7 depicts the deflection of a needle of length $20 \mathrm{~cm}$ embedded in an elastic medium with $P_{\text {input }}, P$, and $Q$ set to $2 \mathrm{~N}, 0.03 \mathrm{~N}$, and $0.3 \mathrm{~N}$, respectively. Fig. 7 provides the deflection of needles for two additional cases with the same tip and insertion forces. In the first case, needle deflection is calculated in the absence of an elastic medium, i.e. $U_{T}=0$. In the second case, needle deflection is computed when there is an elastic medium surrounding the needle but no rupture trajectory, i.e. $U_{T}=\frac{1}{2} \int_{0}^{l_{i}} K_{T} y_{i}^{2} d x$. As seen, the deflection in the absence of a medium is greater than in the presence of the medium, while the absence of a rupture trajectory results in almost no deflection of the needle. For the approximate material and needle properties, the final tip deflection of the needle was iteratively calculated to be $9.2 \mathrm{cms}$, which is in the same range as the observed deflection given in Fig. 3. For this simulation case, the radius of curvature was also observed to be almost constant at $140 \mathrm{~mm}$. The radius of curvature converges after approximately 18 iteration steps.

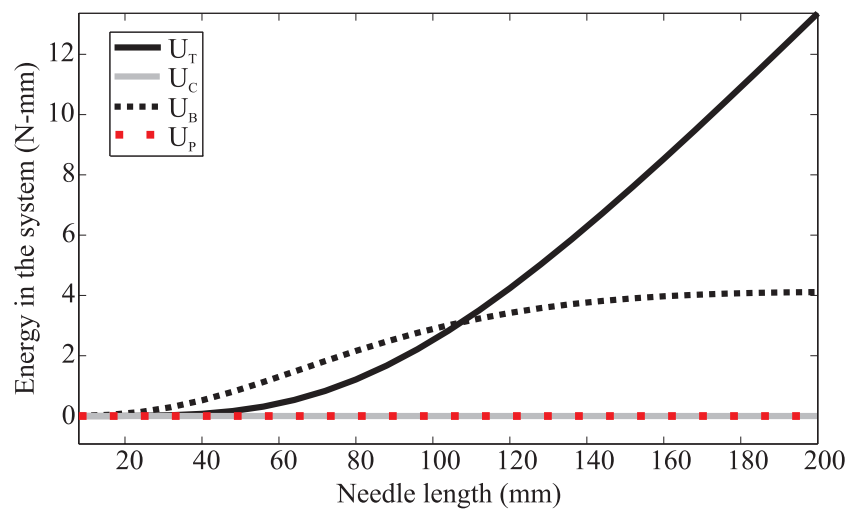

Fig. 8. Contribution of various components of the system energy. 


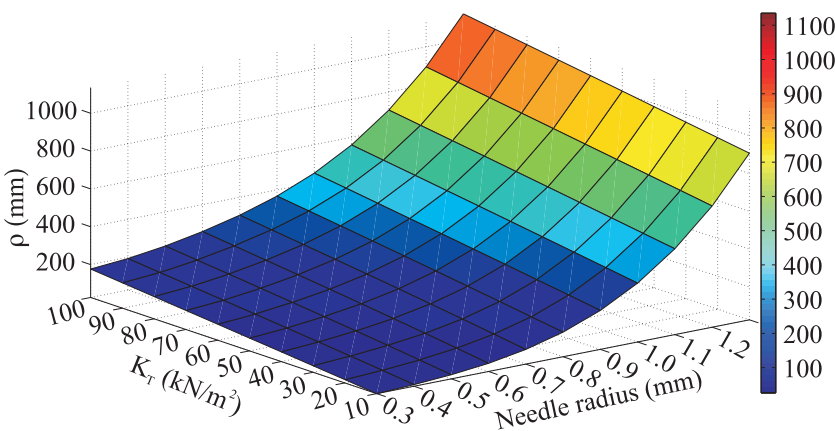

Fig. 9. Variation of the radius of curvature for changes in needle radius and tissue stiffness.

During the initial needle insertion phase, the needle-tissue interaction stiffness does not play a significant role in the needle bending process. But as the needle is further engaged in the elastic medium the importance of the needle-tissue interaction stiffness increases. Fig. 8 shows the contribution of the various energies in the system for a $20 \mathrm{~cm}$ insertion. As seen, $U_{T}$ dominates the total energy contribution to the system. $U_{T}$ and $U_{B}$ contain $76.4 \%$ and $23.5 \%$ of total stored strain energy of the system. Simulation studies were also performed where the needle radius and tissue stiffness per unit length, $K_{T}$, were varied, and the changes in the radius of curvature, $\rho$, were observed. As seen in Fig. 9, for a medium of fixed elasticity, as the radius of the needle increases, the radius of curvature also increases. The numbers for the radius of curvature observed in the simulation studies are in the same range as those seen in the experimental studies given in Table I, but clearly further refinement of material and geometric parameters is needed.

\section{Conclusions}

We developed a mechanics-based model to determine the deflection of a robotically steered needle interacting with a soft elastic medium. The model was influenced by observations made from microscopic images of the needlegel interactions. The simulation results concur with the trend observed in macroscopic observations of a needle inserted into a gel. The results indicated that the energy associated with the needle-tissue interaction stiffness dominates the total potential of the system, and energy due to compression and work done due to rupture and tip loads are less significant.

Building upon our previous work [14], this model accounts for the compression and tear observed in the microscopic images. The energy-based formulation used physically relevant tissue and needle properties, and computes the deflection of the needle by discretizing the needle length into segments. The simulation results for tip deflection are in the same regime as those observed from macroscopic experimental observations of needle insertion. The simulation results for the radius of curvature also converge to a constant value. The method presented could be applied in general to a needle interacting with an elastic medium and are not specific to bevel-tip needles.
Preliminary validation work presented in this study showed that the model and experimental data followed similar trends for needle deflection and constant radius of curvature. The variations in experimental and simulation results could be attributed to: (1) gel properties (both elasticity and toughness) that were not measured accurately, (2) approximate value of the needle insertion force that was provided as input into the simulation model, and (3) mediumspecific bevel tip loads that provided as input to the model. Thus, immediate extension to this work includes detailed validation, which would cover obtaining tissue- and needlespecific material properties, and comparing the results of our model to experimental studies.

\section{REFERENCES}

[1] N. Abolhassani and R. V. Patel, "Deflection of a flexible needle during insertion into soft tissue," in Proc. Int'l. Conf. IEEE Engineering in Medicine and Biology, vol. 1, New York, USA, August/September 2006, pp. 3858-3861.

[2] S. P. DiMaio and S. E. Salcudean, "Interactive simulation of needle insertion models," IEEE Trans. Biomedical Engineering, vol. 52, no. 7, pp. 1167-1179, 2005.

[3] J. A. Engh, G. Podnar, S. Khoo, and C. Riviere, "Flexible needle steering system for percutaneous access to deep zones of the brain," in Proc. 32nd Annual IEEE Northeast Bioengineering Conf., vol. 1, Easton, USA, April 2006, pp. 103-104

[4] D. Glozman and M. Shoham, "Image-guided robotic flexible needle steering," IEEE Trans. Robotics, vol. 23, no. 3, pp. 459-467, 2007.

[5] S. Okazawa, R. Ebrahimi, J. Chuang, S. E. Salcudean, and R. Rohling, "Hand-held steerable needle device," IEEE/ASME Trans. Mechatronics, vol. 10, no. 3, pp. 285-296, 2005.

[6] P. Sears and P. Dupont, "A steerable needle technology using curved concentric tubes," in Proc. IEEE/RSJ Int'l. Conf. on Intelligent Robots and Systems, vol. 1, Beijing, China, October 2006, pp. 2850-2856.

[7] R. J. Webster III, J. S. Kim, N. J. Cowan, G. S. Chirikjian, and A. M. Okamura, "Nonholonomic modeling of needle steering," Int'l. J. Robotics Research, vol. 25, no. 5-6, pp. 509-525, 2006.

[8] N. Abolhassani, R. V. Patel, and M. Moallem, "Needle insertion into soft tissue: a survey," Medical Engineering \& Physics, vol. 29, no. 4, pp. 413-431, 2007.

[9] S. Misra, K. T. Ramesh, and A. M. Okamura, "Modeling of tooltissue interactions for computer-based surgical simulation: a literature review," Presence: Teleoperators \& Virtual Environments, vol. 17, no. 5, pp. 463-491, 2008.

[10] A. M. Okamura, C. Simone, and M. D. O'Leary, "Force modeling for needle insertion into soft tissue," IEEE Trans. Biomedical Engineering, vol. 51, no. 10, pp. 1707-1716, 2004.

[11] O. A. Shergold and N. A. Fleck, "Experimental investigation into the deep penetration of soft solids by sharp and blunt punches with application to the piercing of skin," J. Biomechanical Engineering, vol. 127, no. 5, pp. 838-848, 2005.

[12] R. J. Webster III, J. Memisevic, and A. M. Okamura, "Design considerations for robotic needle steering," in Proc. IEEE Int'l. Conf. on Robotics and Automation, vol. 1, Barcelona, Spain, April 2005, pp. 3588-3594.

[13] S. P. Timoshenko and J. Gere, Theory of elastic stability, 2nd ed. Columbus, USA: McGraw-Hill Companies, 1961.

[14] S. Misra, K. B. Reed, A. S. Douglas, K. T. Ramesh, and A. M. Okamura, "Needle-tissue interaction forces for bevel-tip steerable needles," in IEEE RAS/EMBS Int'l. Conf. on Biomedical Robotics and Biomechatronics, Scottsdale, USA, March 2008, pp. 224-231. 